\title{
Revisiting the cardiovascular risk of hydroxychloroquine in RA
}

\section{Yves-Marie Pers and Guillaume Padern}

\section{Cardiac toxicity can be induced by hydroxychloroquine, especially when used in combination with azithromycin. Interest in hydroxychloroquine and azithromycin as potential therapies for COVID-19 has renewed concerns about the possible cardiovascular risk these drugs present to patients with rheumatoid arthritis.}

Refers to Lane, J. C. E. et al. Risk of hydroxychloroquine alone and in combination with azithromycin in the treatment of rheumatoid arthritis: a multinational, retrospective study. Lancet Rheumatol. https://doi.org/10.1016/S26659913(20)30276-9 (2020).

As a result of the SARS-CoV-2 outbreak, hydroxychloroquine (touted as a potential treatment for COVID-19) has been widely discussed throughout the scientific community. The potential antiviral activity of hydroxychloroquine, which is enhanced when used in combination with azithromycin, has led to renewed interest in this molecule ${ }^{1}$, and the field of rheumatology has found itself at the frontline of the debate as rheumatologists are the foremost prescribers of this drug. Concerns raised about the cardiovascular safety of hydroxychloroquine during trials of the drug for COVID- $19^{2}$ have led researchers to revisit the cardiovascular risks associated with hydroxychloroquine use for other indications, including a large study by Lane et al. ${ }^{3}$ on hydroxychloroquine use by patients with rheumatoid arthritis (RA).

Antimalarial agents, such as hydroxychloroquine, have been prescribed for many years, and their potential uses are still being explored. Hydroxychloroquine is widely used in the treatment of autoimmune diseases such as systemic lupus erythematosus, for which it is effective in preventing relapses and improving survival ${ }^{4}$, and RA, for which it has been used for several decades but has only moderate efficacy ${ }^{5}$. Monotherapy with hydroxychloroquine is not recommended for patients with RA; however, it is sometimes used in combination with methotrexate, as data suggest hydroxychloroquine might potentiate the effects of methotrexate ${ }^{6}$. Although some studies have suggested a vascular protective role for hydroxychloroquine in the context of inflammatory diseases ${ }^{7}$, long-term hydroxychloroquine administration can induce severe cardiac disorders ${ }^{8}$. Hydroxychloroquine provokes sodium and calcium channel blockade, which leads to membrane-stabilizing effects, resulting in conduction disturbances with atrioventricular block, QRS interval widening and QT interval prolongation ${ }^{8}$. Questions have therefore been raised about the safety of hydroxychloroquine, alone or in combination with azithromycin, because of the known tendency of both treatments to lengthen the QT interval and increase the risk of torsade-depointes (a form of polymorphic ventricular tachycardia $)^{2}$.

In patients with RA, there is little robust data about the safety of hydroxychloroquine ${ }^{9}$. For this reason, Lane et al. ${ }^{3}$ studied the effects of hydroxychloroquine use in patients with $\mathrm{RA}$, alone and in combination with azithromycin, in a large-scale ( $n=956,374$ hydroxychloroquine users), international, multicentre, retrospective study. They found that hydroxychloroquine alone, at doses of $\sim 400 \mathrm{mg} /$ day over a 30-day period, had no more adverse effects than sulfasalazine treatment. By contrast, long-term hydroxychloroquine use (anything between 30 days and the theoretical 20-year follow-up) increased cardiovascular mortality compared with sulfasalazine treatment (hazard ratio (HR) 1.65; 95\% CI 1.12-2.44). In the first month of treatment with a combination of hydroxychloroquine and azithromycin, the risks of chest pain or angina and heart failure increased slightly but significantly (HR 1.15; 95\% CI 1.05-1.26 for chest pain or angina and HR 1.22; 95\% CI 1.02-1.45 for heart failure), as did the risk of cardiovascular mortality (HR 2.19; 95\% CI 1.22-3.95) compared with treatment with hydroxychloroquine and amoxicillin ${ }^{3}$.

Although consistent with the described risk of heart rhythm disorders in patients with COVID-19 during treatment with a combination of hydroxychloroquine and azithromycin ${ }^{2}$, the results of the study by Lane et al. ${ }^{3}$ are subject to certain limitations. First of all, the statistical models used were based on data from a variety of databases with heterogeneous populations and different levels of exhaustiveness in data collection, the comparability of which was partially improved by the use of a propensity score. Although this score made it possible to include a very large number of patients in most of the analyses, some of the analyses used smaller samples, such as the calculation of cardiovascular mortality risk for hydroxychloroquine and azithromycin treatment, which used only two databases out of the 14 available. Although propensity scores are intended to homogenize patient characteristics from different databases, the effect of any treatments (related to RA or not) that were used in conjunction with hydroxychloroquine

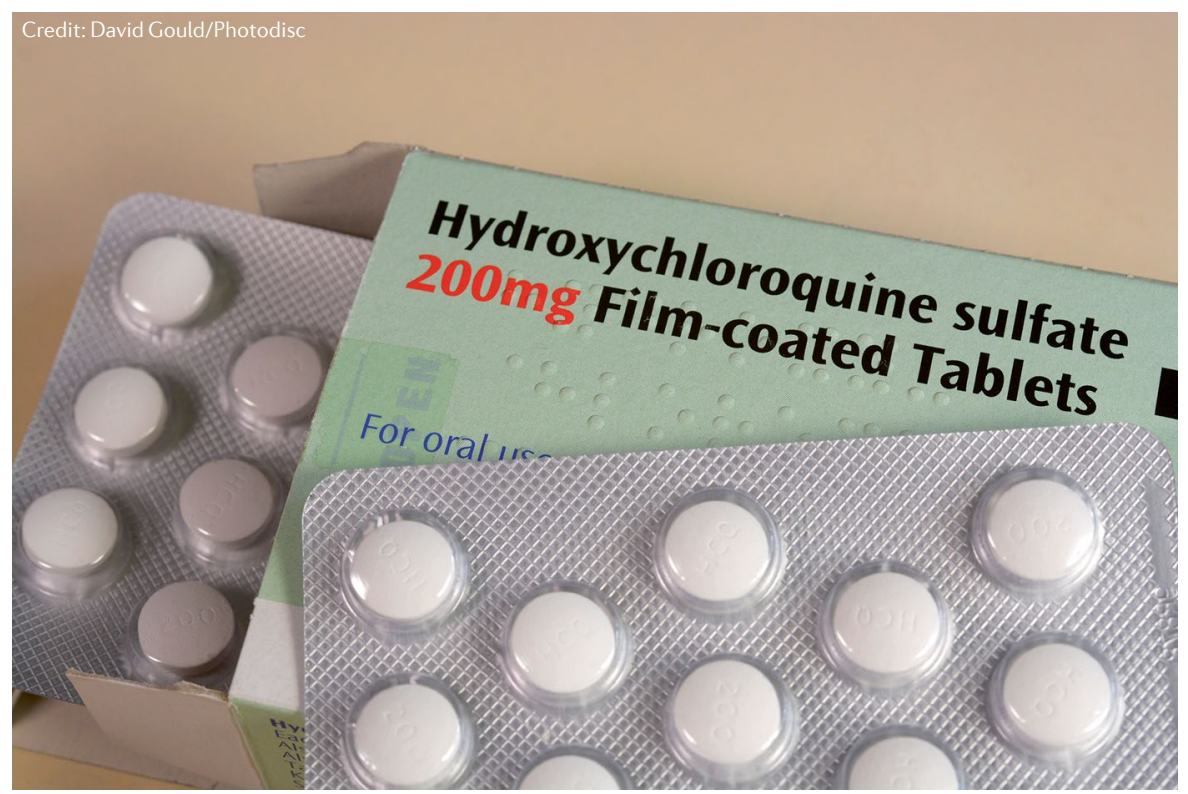




\section{several questions remain about the cardiovascular safety of hydroxychloroquine in the context of RA}

or sulfasalazine could bias the results of this study. Indeed, given that hydroxychloroquine and sulfasalazine are rarely used alone, and the use of therapies can vary from one country to another, it is likely that the precise combinations of therapies differ between patients and could cause confounding bias. Moreover, in the 2019 update to the EULAR recommendations for the management of $\mathrm{RA}^{6}$, hydroxychloroquine does not have exactly the same indication as sulfasalazine, and its use is mainly reserved for patients with mild RA, calling into question the choice of sulfasalazine as a reference treatment by Lane et al. Instead, it might have been preferable to compare patients treated with methotrexate alone or in combination with hydroxychloroquine to those treated with hydroxychloroquine in order to homogenize the study population and avoid the bias associated with adverse events of the reference treatment. In addition, it was quite unexpected that Lane et al. ${ }^{3}$ noted an increase in cardiovascular mortality without a concurrent increase in heart failure or cardiac arrhythmias, the two main conditions involved in the pathophysiology of hydroxychloroquine toxicity ${ }^{8}$. The mechanisms explaining this excess mortality thus seem to be poorly understood.

Several questions remain about the cardiovascular safety of hydroxychloroquine in the context of RA, including questions around the lack of a dose-response effect and the unknown association with disease activity, given that more cardiovascular events would be expected if RA was not well controlled. Moreover, the time period used to study the effect of long-term use of hydroxychloroquine on cardiovascular events by Lane et al. ${ }^{3}$ was relatively brief ( $\sim 1$ year of hydroxychloroquine use) compared with the previously published reports of hydroxychloroquine toxicity ${ }^{8}$. Indeed, the accumulation of metabolic products (glycogen and phospholipids) in cardiomyocytes as a result of dysfunctional lysosomal enzymes seems to be a slow process that usually requires a high cumulative dose of an antimalarial and a long treatment duration (median 8 years) $)^{10}$.

To conclude, although hydroxychloroquine seems to be generally well tolerated by patients with RA, cardiac toxicity is a rare and severe iatrogenic adverse effect. Further studies are required to better explore this cardiac toxicity and its importance. Because of the variability and non-specificity of hydroxychloroquine-associated cardiac toxicity, accurate diagnosis is difficult, especially in patients with RA, who often have cardiovascular comorbidities. The results of the study by Lane et al. ${ }^{3}$ will probably not change our prescribing practices for RA, but they should encourage the cautious use of hydroxychloroquine, especially in combination with azithromycin, in those patients who also have chronic heart disease. Indeed, given the increased risks of QT interval prolongation and torsade-de-pointes, it seems sensible to advocate for close electrocardiographic monitoring before hydroxychloroquine initiation, one month after and then every 2 years, including a clinical-morphological follow-up to determine early cardiac failure ${ }^{8}$.
Yves-Marie Pers ${ }^{\bowtie}$ and Guillaume Padern

INSERM U1183, Institute for Regenerative Medicine and Biotherapy, $\mathrm{CHU}$ Montpellier and University of Montpellier, Montpellier, France.

凶e-mail:ym-pers@chu-montpellier.fr https://doi.org/10.1038/s41584-020-00521-x

1. Gautret, P. et al. Hydroxychloroquine and azithromycin as a treatment of COVID-19: results of an open-label non-randomized clinical trial. Int. J. Antimicrob. Agents 56, 105949 (2020).

2. Mercuro, N. J. et al. Risk of QT interval prolongation associated with use of hydroxychloroquine with or without concomitant azithromycin among hospitalized patients testing positive for coronavirus disease 2019 (COVID-19). JAMA Cardiol. 5, 1036-1041 (2020).

3. Lane, J. C. E. et al. Risk of hydroxychloroquine alone and in combination with azithromycin in the treatment of rheumatoid arthritis: a multinational, retrospective study. Lancet Rheumatol. https://doi.org/10.1016/ S2665-9913(20)30276-9 (2020).

4. Ruiz-Irastorza, G. et al. Clinical efficacy and side effects of antimalarials in systemic lupus erythematosus: a systematic review. Ann. Rheum. Dis. 69, 20-28 (2010).

5. Rempenault, C. et al. Clinical and structural efficacy of hydroxychloroquine in rheumatoid arthritis: a systematic review. Arthritis Care Res. 72, 36-40 (2020).

6. Smolen, J. S. et al. EULAR recommendations for the management of rheumatoid arthritis with synthetic and biological disease-modifying antirheumatic drugs: 2019 update. Ann. Rheum. Dis. 79, 685-699 (2020).

7. Rempenault, C. et al. Metabolic and cardiovascular benefits of hydroxychloroquine in patients with rheumatoid arthritis: a systematic review and meta-analysis. Ann. Rheum. Dis. 77, 98-103 (2018).

8. Chatre, C. et al. Cardiac complications attributed to chloroquine and hydroxychloroquine: a systematic review of the literature. Drug Saf. 41, 919-931 (2018).

9. Sepriano, A. et al. Safety of synthetic and biological DMARDs: a systematic literature review informing the 2019 update of the EULAR recommendations for the management of rheumatoid arthritis. Ann. Rheum. Dis. 79 760-770 (2020).

10. Homewood, C. A. et al. Lysosomes, $\mathrm{pH}$ and the anti-malarial action of chloroquine. Nature $\mathbf{2 3 5}$, 50-52 (1972).

\section{Competing interests}

The authors declare no competing interests. 